\title{
BMJ Open Nature-based care opportunities and barriers in oncology contexts: a modified international e-Delphi survey
}

\author{
Sarah Blaschke, ${ }^{1,2}$ Clare C O'Callaghan, ${ }^{1,3,4}$ Penelope Schofield ${ }^{1,2,5}$
}

To cite: Blaschke S, O'Callaghan CC, Schofield P. Nature-based care opportunities and barriers in oncology contexts: a modified international e-Delphi survey. BMJ Open 2017;7:e017456. doi:10.1136/ bmjopen-2017-017456

- Prepublication history and additional material for this paper are available online. To view these files please visit the journal (http://dx.doi.org/10. 1136/bmjopen-2017-017456).

Received 27 April 2017 Revised 8 August 2017 Accepted 9 August 2017

\section{CrossMark}

${ }^{1}$ Department of Cancer Experiences Research, Peter MacCallum Cancer Centre, Melbourne, Victoria, Australia ${ }^{2}$ Sir Peter MacCallum Department of Oncology, Faculty of Medicine, Dentistry and Health Sciences, The University of Melbourne, Parkville, Victoria, Australia

${ }^{3}$ Department of Medicine, St Vincent's Hospital, The University of Melbourne, Melbourne, Victoria, Australia

${ }^{4}$ Palliative Care Service, Cabrini Health, Melbourne, Victoria, Australia

${ }^{5}$ Department of Psychology, Faculty of Health Sciences, Swinburne University of Technology, Melbourne, Victoria, Australia

Correspondence to

Sarah Blaschke;

sarah.blaschke@petermac.org

\section{ABSTRACT}

Objective To develop recommendations regarding opportunities and barriers for nature-based care in oncology contexts using a structured knowledge generation process involving relevant healthcare and design experts. Design Four-round modified electronic Delphi study. Oncology patients' nature-based recommendations, uncovered in preceding qualitative investigation, were included in the first round for the expert participants' consideration. Key items (opportunities and barriers) were developed using data aggregation and synthesis, followed by item prioritisation and 10-point Likert scale ranking (1=not important, 10=very important). Descriptive statistics were calculated to assess items of highest importance representing expert recommendations.

Context Online Delphi process constituting an electronic international survey.

Participants A purposive sample of 200 potential panellists (recruitment target $n=40$ ) comprising healthcare practitioners, managers, designers, architects and researchers were invited to participate; experts were identified via research networks, snowballing and systematic literature review.

Results 38 experts across seven countries (Australia, USA, UK, New Zealand, Canada, Denmark and Sweden) returned questionnaire 1 , which determined consent and acceptance for participation. Initial response rate was $19 \%$, and subsequent response rates were $84 \%, 82 \%$ and $84 \%$ for rounds 2, 3 and 4, respectively. The Delphi panel developed recommendations consisting of 10 opportunities and 10 barriers. The following opportunities were rated to be of highest importance: window views from clinical areas onto nature; outdoor settings, gardens and courtyards with easy and effortless access; and naturebased physical exercise adapted to patient requirements. Highest-rated barriers for nature-based oncology care included lack of knowledge and awareness about benefits of nature engagement and inaccessibility, not considering access requirements for the very sick and frail.

Conclusions Experts suggested and agreed on a set of recommendations, which represent critical considerations for the safe adoption of nature-based oncology opportunities. These findings fill a gap in understanding about helpful nature-based oncology care and may translate into oncology design and innovation.

\section{INTRODUCTION}

There is growing interest internationally in the potential health benefits of exposure

\section{Strengths and limitations of this study}

- An integrated validation process gave panellists opportunity to request revision of their input as required for accuracy and reliability of interpretations.

- The international and cross-disciplinary Delphi panel contributed breadth of expert input to inform new understanding about helpful nature engagement in oncology contexts.

- The e-Delphi structures a collaborative feedback process allowing experts across different geographic locations to anonymously input knowledge and experience.

- The findings represent contributions from a specific expert sample and may not be reproducible when involving other experts.

to, and engagement with, nature. Public and academic debate around incorporating nature-based opportunities in healthcare setting and service design is increasing. This is partly due to a growing research evidence base showing beneficial interchanges between health, well-being and nature exposure for the general public $^{12}$ and specific disease populations. $^{34}$

Cancer will soon affect at least one in three people either personally or through a relative or friend. ${ }^{5}$ One-third of lives lost to cancer are attributable to behavioural and lifestyle choices, and $30 \%$ of these cancer deaths are preventable by attending to key risk factors. ${ }^{5}$ Therefore, access to health-centric care strategies, which promote positive health behaviour and prevent poor lifestyle choices, can alleviate growing healthcare burden and support those affected by cancer. Public health promotion research has identified contact with nature as an underused health resource, ${ }^{6}$ which may translate into new avenues for supportive oncology services and setting design: 'contact with nature may offer an affordable, accessible and equitable choice on tackling the imminent epidemic, with both preventive and restorative [public] health strategies'. 
Such health-centric strategies engage a person's own capacity to self-manage health and disease, ${ }^{7}$ which is relevant for oncology patients who may be exposed to unavoidable biopsychosocial stressors in clinical as well as in daily living environments. Receiving a cancer diagnosis and undergoing treatment can cause high levels of psychological distress across the continuum of the disease. ${ }^{8}$ Patients with cancer may face numerous physical, psychological and economic challenges and experience unmet psychosocial needs related to, for example, employment, social engagement, close relationships, sleep quality and sexual activity. ${ }^{8} 10$ A Canadian survey with 913 patients with cancer showed that $94 \%$ of patients experience one or more of the following quality-of-life-related symptoms: fatigue, anxiety, depression and sleep disturbance. ${ }^{10}$ Preliminary research has also shown various biopsychosocial benefits from exposure to, and engagement with, nature when living with cancer, including improved quality of life,${ }^{11}$ increased positive health behaviour such as physical exercise and fruit and vegetable consumption, ${ }^{12}$ restored attention ${ }^{13}$ and increased social engagement. ${ }^{14}$ Furthermore, patients with cancer have reported to value opportunities to engage with nature when navigating the ongoing challenges of cancer diagnosis and treatment by using nature as a physical and psychological resource for restorative processes and normalisation. ${ }^{15}$ Although there is interest in nature's role in supporting patients with cancer from a preventative and rehabilitative perspective, there is a gap in research on nature's role in clinical settings, which provide patient care. Refining our understanding about what patients value becomes relevant for healthcare policy if the aim is to maintain or even improve patient well-being and quality of life during and beyond cancer treatment. This shift towards patient-centred care demands more comprehensive regard of patients' personal challenges and wide-ranging experiences related to health and recovery. Effective healthcare design thus requires rigorous investigation to respond sensitively to such patient-reported values and to ensure responsible use of limited resources. Patients' health and recovery requirements stand at the forefront of this complex dialogue, which includes multiple stakeholders involved in healthcare design and innovation.

To develop recommendations for nature-based oncology care, a synthesis of opinion from a range of experts is required. This Delphi study solicited input from relevant healthcare and design experts drawn from a range of professional and academic roles (including cancer-specific experts) and explored their critical input important for the provision of beneficial and safe nature engagement in oncology contexts. Following a systematic literature ${ }^{16}$ review, to our best knowledge, this is the first collection and synthesis of expert opinion on this topic across healthcare and design disciplines.

\section{OBJECTIVE}

The objective was to develop recommendations related to opportunities and barriers for nature-based care in oncology contexts using a structured knowledge generation process involving relevant healthcare and design experts.

\section{STUDY DESIGN}

The present study is part of a $\mathrm{PhD}$ project and follows from qualitative research into the use of nature of patients with cancer and its relevance in their experiences of health and recovery. ${ }^{15}$ From this, patients' own recommendations were extracted and included in the present investigation for healthcare experts' consideration when developing their own views about integrating nature into oncology.

The full study protocol has been published elsewhere ${ }^{17}$ and describes in detail the Delphi methodology adopted in this research project. The main method modification made to suit the present study was to use a three-phase sequence ${ }^{18}$ with the aim to structure an iterative feedback process with a predetermined number of four questionnaires (rounds) rather than following the traditional method of using as many questionnaires as needed to reach strict consensus. Strict consensus was not the aim of this study. Phase 1 comprised two questionnaires. Questionnaire 1 (Q1) served idea generation and aimed to uncover salient issues (items) with relevance to the topic by inviting panellists to brainstorm and open-endedly contribute their ideas. Panellists were provided with patient-reported recommendations and cautions for nature-based care from previous research. Responses were categorically aggregated and recirculated to the panel who were given opportunity in questionnaire 2 (Q2) to verify cogent interpretation of their data. Next, panellists short-listed items of priority, which were subsequently ranked 1 to 10 reflecting the relative degree of consensus among the panel about the items' importance. Questionnaires were electronically administered via email and were planned to take a minimum of 4 weeks, respectively ${ }^{19}$ : 2 to 3 weeks for panellists to respond (including reminder emails prior to round closing deadlines) and 1 week to analyse response data and draft the next questionnaire. Figure 1 summarises and illustrates the study design. The protocol ${ }^{17}$ and related study materials were designed following the SPIRIT 2013 Checklist $^{20}$ where appropriate.

\section{Definition of 'expert'}

Delphi studies use various definitions to identify and include relevant experts such as 'informed advocates', ${ }^{21}$ 'informed individuals', 'specialist in their field' or persons with 'knowledge about a specific subject'. ${ }^{22}$ Central to these formulations is the description of individuals who possess both knowledge and experience, which enables them to articulate informed opinion and provide relevant 
Relevant experts identified:

- healthcare practitioners

- healthcare management

- healthcare designers

- healthcare \& design academics

Recruitment target $n=40$
Strategy 1 key authors identified via systematic literature review

Strategy 2 purposive sampling via researchers' networks

Strategy 3 ongoing snowballing via networks and contacted experts

Questionnaire 1 generated based on preceding qualitative investigation

Questionnaire 1 pilot tested with

2 - 3 researchers

200 experts invited via email:

- attached project description

- attached Questionnaire 1

- return of Questionnaire 1 is considered consent

Questionnaire 1 adapted according to feedback

PHASE 1

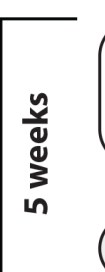

Questionnaire 1 ( $n=38)$

- Experts consider summary of cancer patients' recommendations

- Experts generated 455 items: 250 Opportunities, 205 Barriers

Questionnaire 2 designed based on Questionnaire 1 analyses
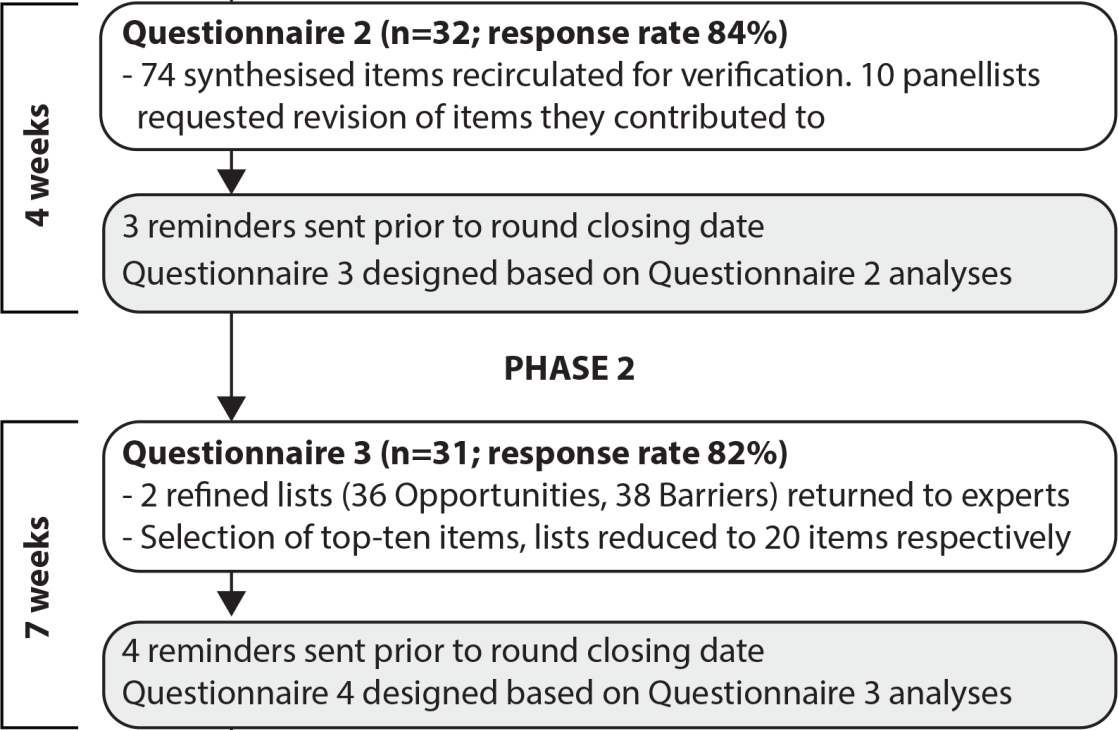

Questionnaire 3 ( $n=31$; response rate $82 \%$ )

- 2 refined lists (36 Opportunities, 38 Barriers) returned to experts - Selection of top-ten items, lists reduced to 20 items respectively

4 reminders sent prior to round closing date

Questionnaire 4 designed based on Questionnaire 3 analyses
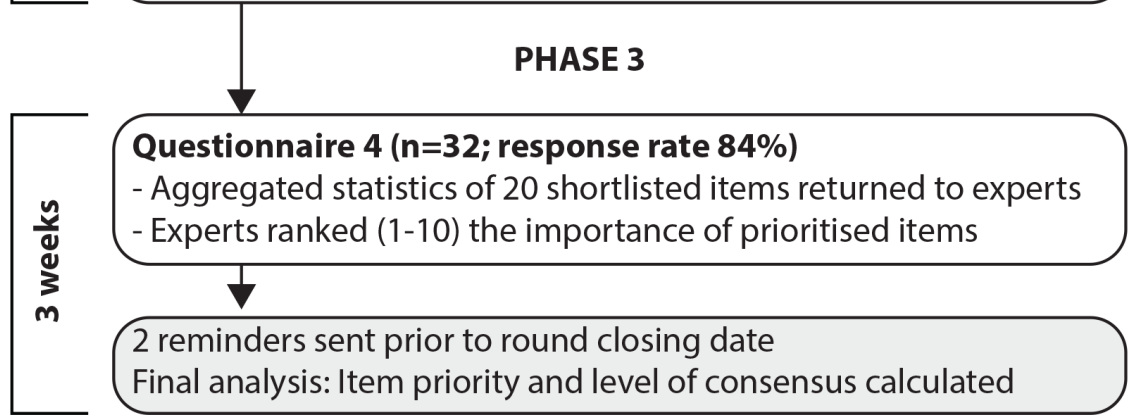

Figure 1 Study flow chart. 
input about a given topic. This definition of an expert was adopted by the present study.

\section{Composing the Delphi panel}

This study aimed to compose a heterogeneous panel of experts to bring a range of disciplinary viewpoints to the surface and articulate great complexities about the topic. Five groups of diverse yet relevant stakeholders were identified in the area of cancer care innovation: (1) patients with cancer, (2) healthcare practitioners (HPs), (3) healthcare management (HM), (4) healthcare architects and designers (ADs) and (5) and healthcare and design academics. The panel did not include additional patients with cancer. The rationale for this composition was, first, that the present study was built on a substantial amount of data already collected from patients with cancer in the preliminary qualitative study. ${ }^{15}$ Second, this current study focused specifically on the views of those who are involved in healthcare development to ascertain feasible nature engagement opportunities in oncology contexts and their realistic limitations.

\section{Sample size}

Delphi studies use varying panel sizes ranging from single digits to low hundreds. ${ }^{21}$ Seven is considered an acceptable minimum panel size with accuracy rapidly declining as the number becomes smaller. ${ }^{21}$ It is understood that the levels of census among experts are of more interest than the power of frequencies of response. ${ }^{18}{ }^{23}$ Two hundred eligible experts were invited with the intention to recruit 10 participants per expert group, and the total target was 40 experts.

\section{Recruitment procedure}

To collect input from a diverse range of international healthcare and design experts involved in cancer care innovation, three sampling strategies were devised to identify relevant, eligible participants: (1) purposive list building (research networks), (2) snowballing and (3) systematic literature review to identify key authors. The following predefined inclusion criteria previously used in Delphi panel recruitment ${ }^{19}$ supplemented the selection procedure: (1) capable of contributing relevant input (knowledge and experience), (2) willingness and sufficient time to complete all four rounds and (3) sufficient English skills to communicate ideas effectively.

Experts were contacted with an introductory email containing Q1 and information about participation to explain the required commitment. Experts accepted study participation and membership to the Delphi panel by completing and returning Q1, which included a demographics section. Ethics approval was gained from the Peter MacCallum Cancer Centre Human Research Ethics Committee (LNR/16/PMCC/65). Experts were assured of confidentiality and that their identities were only known to the research team throughout the study period and that no identifiable information would be presented in final reporting of the study.

\section{METHODS}

The Delphi method is an established research tool for complex problem solving, which solicits expert opinion through a structured, iterative process. ${ }^{18}$ The present study aimed to elicit and synthesise diverse disciplinary viewpoints to guide the development of expert recommendations. The modified electronic Delphi method fits this purpose and provides following key advantages: (1) serves the dual purpose of soliciting broad expert opinion followed by priority ranking ${ }^{24}$; (2) can conclude at a predefined number of rounds because strong consensus is not required when degrees of agreement and group attitudes are of interest ${ }^{21} ;(3)$ structures a rigorous and rapid feedback-based (online) communication process ${ }^{25}$; (4) frees communication from logistical challenges, peer pressure and 'group-think' scenarios ${ }^{26}$ and (5) cross-pollinates multidisciplinary expertise achieving broader understanding than would be reached from a single discipline alone. ${ }^{24}$

\section{Procedure and data analysis}

Delphi's primary research tool is a series of iterative questionnaires built from participants' stepwise input. The research team designed Q1, and Q2-Q4 were subsequently created to reflect content of responses from the ongoing data collection and analysis. Following ethics approval, data were collected from June 2016 to October 2016. As required, up to four email reminders were sent in weekly intervals for Q2, Q3 and Q4. A brief description of each questionnaire follows. Detailed overview and illustrations of the administered questionnaires can be found in the published study protocol. ${ }^{17}$

\section{Q1: idea generation}

Q1 collected the following demographic information: name, contact details, description of professional role, years served in role of expertise and country of professional residence/affiliation. Two sections then followed. Section A constituted a summary of the anonymised recommendations of patients with cancer, extracted from the preceding qualitative investigation, ${ }^{15}$ which expert participants were invited to consider. Section B queried experts' own recommendations for nature-based care opportunities in the oncology setting and asked for factors they perceived are barriers to their provision. Q1 was pilot tested by two psycho-oncology researchers unfamiliar with the Delphi method, who were asked to provide feedback about their question-and-answer process when completing the questionnaire. ${ }^{27}$ This process ensured Q1 was comprehensible to naive Delphi responders and that the intended scope and quality of response were achieved. All data were entered and managed in Microsoft Excel. The analysis first removed identical responses, then the remaining ideas were collated, synthesised and edited to achieve consistent terminology of items expressing similar ideas and, finally, comparable items were grouped into categories. An inter-rater process constituting the three authors ( $\mathrm{SB}, \mathrm{CO}$ and $\mathrm{PS}$ ) 
assisted interpretative congruity as recommended for thematic analysis. ${ }^{28}$

\section{Q2: validation of categorised items}

Q2 was designed based on responses from Q1 and aimed to strengthen construct validity ${ }^{24}$ according to the concept of 'member checking'. ${ }^{28}$ All categorised items were recirculated to all experts for verification. Based on responses, items were further again subjected to inter-rater discussion, refined and finalised when agreement was reached.

\section{Q3: prioritising items}

Q3 included a structured format listing all items generated thus far in random arrangement to minimise response bias. Each panellist selected 10 items (top 10) from each list (opportunities and barriers). Items selected by the majority of experts were aggregated representing a majority vote. Lists were reduced according to the importance of items calculated based on the sum of points allocated by each expert to their top 10 items, that is, item ' 1 ' indicating highest importance was coded with 10 points, item '2' coded with nine points and so on. To avoid overburdening panellists, ${ }^{18}$ the target size of total items for the final round was no more than 40 items (20 opportunities and 20 barriers).

\section{Q4: ranking items}

Q4 was designed to elicit levels of consensus (not achieve consensus) in the ranking of relevant items. This questionnaire included aggregated statistical group responses generated for each included item thus far. Each panellist submitted a rank ordering of the items for each of the condensed lists (opportunities and barriers) using corresponding 10-point Likert-type scales (1=notimportant at all, 10=veryimportant). Statistical analyses were performed using IBM SPSS V.23 for Macintosh. ${ }^{29}$ Descriptive statistics (median scores and $\mathrm{IQR}$ ) were calculated for the entire panel to indicate items' relative importance. Differences in consensus among subgroups were explored. Dual-role occupants were assigned to both groups they hold expertise in. Consensus was defined as $75 \%$ agreement. $^{30}$

\section{RESULTS}

\section{Panellists}

\section{Characteristics of Delphi panellists}

Two hundred potential panellists were identified and sent an invitation to participate, which included Q1. Thirty-eight questionnaires (19\%) were returned. The participant characteristics are shown in table 1. Responders represented seven countries: Australia (19), USA (8), UK (3), New Zealand (2), Canada (2), Denmark (3) and Sweden (1). Twenty-three responders (61\%) were designated into one of the four expert groups; 15 (38\%) occupied dual roles and were designated into two groups. Overall, participants reported high levels of expertise with an average of 20.5 years (SD 10) of experience in their respective roles. Combined, this expert sample $(n=38)$ represents 780 years of accumulated expertise.

\section{Phase 1: generation and validation of items}

Panellists contributed a wealth of descriptively rich data in Q1 (idea generation). In total, they made 250 separate suggestions for opportunity items and 205 suggestions for barrier items. Further analysis identified 55 unique items (35 opportunities, 20 barriers) after removing identical suggestions, which expressed the same ideas in different words. The condensed items were grouped into 19 categories (see box), which emerged during the analytic process. Each item was assigned anonymous identifiers corresponding with panellists who contributed to the item. In Q2 (validation of categorised items), 11 panellists requested revision of their items when asked for verification of the synthesised data. Data interpretation and description were refined based on panel feedback received.

\section{Phase 2: prioritisation of items}

Thirty-one panellists $(82 \%)$ selected their priority items (top 10) in Q3 representing a majority vote of items of highest importance. Forty items achieving the highest rankings were short-listed (20 opportunities and 20 barriers) and included in Q4 for final importance rating (see online supplementary appendix 1 for detailed findings).

\section{Phase 3: ranking of items}

\section{Highest-ranked items in Q4 $(n=32 ; 84 \%)$}

Not all 32 panellists who returned $Q 4$ completed ratings of all items; some items were missed, and in some instances, two numbers were indicated on the 10-point scale. These were excluded from the analysis. Thirty-two ratings were available for 25 of the Q4 items, 14 items were given a minimum of 30 ratings and only 1 item received 29 ratings from panellists who returned Q4 $(n=32)$. The distribution of scores was negatively skewed, with an average of $82 \%$ panellists assigning scores $\geq 7$ Likert scale points. However, the level of consensus (75\% agreement) among panellists on whether an item was important (score $\geq 7$ ) ranged from $48 \%$ to $100 \%$.

Thirty of the 40 rated items met the consensus level of receiving a score of $\geq 7$ points from $\geq 75 \%$ of respondents (see online supplementary appendix 1 for details). The 10 highest-rated opportunity items and barrier items and the levels of consensus achieved are presented in table 2. These final items represent the panel's list of recommendations.

The highest-rated opportunity item was 'Window views from clinical areas onto nature, garden, sea, sky, weather, people watching, greenery, trees, outside world, daylight, night sky, escape, movement, change, without glare, attention to privacy (one-way views)' (median $=10 ; 97 \%$ consensus); the highest-rated barrier item was 'Building design and site constraints, missed opportunities: Layout, building orientation, surrounding views, lack of available 
Table 1 Characteristics of Delphi panellists

\section{Expertise, $\mathrm{n}=38$,}

years combined $Q 1, n=38 \quad Q 2, n=32 \quad Q 3, n=31 \quad Q 4, n=32$

\begin{tabular}{|c|c|c|c|c|c|}
\hline \\
\hline \multicolumn{6}{|l|}{$\begin{array}{l}\text { Healthcare practitioner (HP). Fields included specialist } \\
\text { nursing, medical oncology, palliative care, clinical } \\
\text { psychology, physiotherapy, art therapy and horticultural } \\
\text { therapy }\end{array}$} \\
\hline $\begin{array}{l}\text { Expert groups } \\
\text { Healthcare practitioner (HP). Fields included specialist } \\
\text { nursing, medical oncology, palliative care, clinical } \\
\text { psychology, physiotherapy, art therapy and horticultural } \\
\text { therapy } \\
\text { Healthcare management (HM) }\end{array}$ & \multirow{2}{*}{$\begin{array}{l}25 \\
64\end{array}$} & \multirow{2}{*}{$\begin{array}{l}1 \\
3\end{array}$} & \multirow{2}{*}{$\begin{array}{l}1 \\
2\end{array}$} & \multirow{2}{*}{$\begin{array}{l}0 \\
3\end{array}$} & 1 \\
\hline $\begin{array}{l}\text { Healthcare management }(\mathrm{HM}) \\
\text { Healthcare architects and designers (AD) }\end{array}$ & & & & & 2 \\
\hline $\begin{array}{l}\text { Academics (A). Fields included occupational therapy, } \\
\text { community health, horticultural therapy, medical, psycho- } \\
\text { oncology, health psychology, healthcare design, palliative } \\
\text { and end-of-life care }\end{array}$ & 253 & 11 & 10 & 8 & 8 \\
\hline
\end{tabular}

Dual roles

\begin{tabular}{|c|c|c|c|c|c|}
\hline$A, A D$ & 86 & 4 & 3 & 4 & 4 \\
\hline $\mathrm{A}, \mathrm{HP}$ & 121 & 6 & 5 & 6 & 6 \\
\hline $\mathrm{A}, \mathrm{HM}$ & 22 & 1 & 1 & 1 & 1 \\
\hline$A D, H P$ & 47 & 2 & 2 & 1 & 1 \\
\hline HP, HM & 28 & 2 & 2 & 2 & 2 \\
\hline \multicolumn{6}{|c|}{ Geographic location } \\
\hline Australia & & 19 & 16 & 15 & 17 \\
\hline USA & & 8 & 8 & 8 & 7 \\
\hline UK & & 3 & 2 & 2 & 2 \\
\hline New Zealand & & 2 & 1 & 2 & 1 \\
\hline Canada & & 2 & 2 & 1 & 2 \\
\hline Denmark & & 3 & 2 & 2 & 2 \\
\hline Sweden & & 1 & 1 & 1 & 1 \\
\hline
\end{tabular}

$33 / 38(87 \%)$ panellists completed questionnaire 2,31 (82\%) completed questionnaire 3 and 32 (84\%) returned questionnaire 4 . In total, three participants formally withdrew participation; other non-responders did not give reasons for discontinuing participation.

space were not considered in planning and development phase' (median $=9 ; 91 \%$ consensus).

\section{Differences in expert group opinion}

Experts' views about the topic converged rather than diverged as evidenced by the distillation of 455 separate suggestions into 55 key 'opportunities' and 'barriers'. Essentially, similar deep concerns were expressed through experts' parallel suggestions for opportunities and barriers. This was further shown in the final Likert scale results, which achieved similar high and low appraisals of items across expert groups (see online supplementary appendix 1 for median scores and IQR by subgroup). The only small variation in ratings was found in how the 'healthcare management' group rated the Barrier items, that is, the selection of Barrier items judged to be of highest and lowest importance. In contrast to the other three expert groups, this group appraised the following Barrier items: 7G (median=9.5), 8B (median=9.5) and 10F (median $=9.5$ ) to be of highest importance and $1 \mathrm{~A}$ (median=7) and 4C (median=7) to be of least importance. However, due to the small number of experts in this group $(n=4)$, no meaningful inferences can be made about a general opinion deviation among the expert groups.

\section{DISCUSSION}

This modified e-Delphi study involved four groups of international experts involved in cancer care innovation and aimed to systematically identify and articulate realistic opportunities and important barriers most relevant for the provision of nature-based care in oncology contexts. Contributions to item generation in Q1 were extensive and of high quality; an acceptable panel size was achieved, and participant retention was high for all four rounds. Allowing panellists in Q1 to reflect on patients' own recommendations increases the usefulness of findings and their relevance for patients. An integrated validation process gave panellists opportunity to request revision of their input as required for correctness and strengthened reliability of interpretations and improved the validity of findings. ${ }^{24}$ 
Box Categories and number of validated items generated by the Delphi panel in Q1 $(n=38 ; 19 \%)$ and $Q 2(n=33 ; 87 \%)$

\author{
Categories (number of items grouped into each category) \\ Opportunities \\ - A. Accessibility. To enable access to nature engagement (5) \\ - B. Clinical appropriateness and safety (2) \\ - C. Design. To enable optimal multisensorial connection with nature \\ (12) \\ - D. Education and awareness. To enable the context for nature \\ engagement (3) \\ - E. Physical exercise, sensory stimulation, aesthetic experience (2) \\ F. Potential nature-based activities (9) \\ - G. Repair and maintenance (3) \\ Barriers \\ - A. Building and site constraints (3) \\ B. Champion unavailable (1) \\ C. Cost and lack of resources (4) \\ D. Inaccessibility (3) \\ - E. Inadequate knowledge and lack of awareness about existing \\ knowledge (9) \\ - F. Inappropriate design choices and execution (4) \\ - G. Incompatible with current healthcare paradigm (2) \\ - H. Individual preferences—one 'design' does not fit all (1) \\ I. Negative trigger (1) \\ - J. Patient burden, limitations and underutilisation (4) \\ K. Patient perspective not included (2) \\ L. Risk and safety (4)
}

Note: Some categories reflect how panellists connected process and outcome, for example, educating stakeholders about available nature-based modalities and benefits (opportunity D, process) may mitigate inadequate knowledge and awareness (barrier $\mathrm{E}$, outcome).

Although numerous opportunities for nature exposure and nature engagement were identified, several patient-centric insights arose as central concepts in the panel's thought process. The panel recognised multiple patient needs and preferences, which could be addressed through nature-based elements in the care setting. Among these were patients' need for connection with the outside world (1C), accessible retreat from clinical scenarios (2A), physical exercise opportunities (3E), contemplative and solitary experiences $(6 \mathrm{C})$ as well as socially engaging and communal experiences $(9 \mathrm{~F})$ and aesthetically and sensory enriching (10C) yet climatically comfortable and safely manoeuvrable environments $(8 \mathrm{C}$ and $4 \mathrm{~B}$ ). These findings connect with existing literature confirming a similar broad range of desired patient experiences when dealing with cancer diagnosis; they include, for example, contact with the outside world through window views, ${ }^{11}$ social experiences and engaging in peer activities, ${ }^{31}$ seeking respite from clinical reality, ${ }^{32}$ adopting new physical activities for improving fitness and well-being, ${ }^{33}$ stimulating and aesthetic experiences that enliven the physical senses ${ }^{32} 34$ and contemplating and connecting meaningfully with oneself and notions of spirituality. ${ }^{35}{ }^{36}$ In related research, it was found that patients in the ICU, in particular long-stay patients, can benefit from nurse-facilitated nature-based interventions to enhance the clinical environment for better patient outcomes and to avoid unnecessary environmental stressors. ${ }^{37}$ Examples of recommended nature-based opportunities available in ICU nursing include positioning patient beds to view the outside world, appropriate natural light exposure, taking patients to outside areas when able to mobilise and providing nature-based soundtracks and pictures of nature in the patient room. ${ }^{37}$ Our findings further connect with research investigating other vulnerable patient groups such as patients with advanced dementia. Fleming and colleagues ${ }^{38}$ confirm the effective role of environmental design in the care of this patient group. Their survey study shows strong agreement among healthcare experts about the importance of certain characteristics of care environments, which include: access to the outdoor and indoor nature (plants, natural light and fresh air), design that promotes sensory and social engagement and opportunities for privacy, safety and security. ${ }^{38}$

Additionally, the panel in the present study raised staff concerns about the importance of designing for staff privacy and workplace exercise opportunities, which confirms related research evaluating outdoor settings in a paediatric cancer centre that included the benefits for staff usage ${ }^{14}$ Importantly, category C (Design. To enable optimal multisensorial connection with nature) emerged as a prominent finding, which highlights the central role of architecture and design of cancer care environments and its central role in shaping the breadth and quality of experiences available to the various users. This was also found in research on the effects of environmental features in oncology settings. ${ }^{39}$ Further, the findings elucidated experts' important recommendations to educate healthcare teams about the value, benefit and practical implementation of nature-based opportunities and the importance of professional and properly budgeted maintenance of any integrated nature features. This vital education and funding component relies strongly on available and accessible evidence, which supports the value and usefulness of allocating healthcare resources to nature-based initiatives in healthcare settings. ${ }^{40}$

Factors deemed as barriers to the provision of naturebased elements spanned a wide spectrum of collective concerns among the experts including, for example, building constraints (1A), inappropriate and inauthentic design choices ( $5 \mathrm{~F}$ and $10 \mathrm{~F})$, lack of awareness and ongoing advocacy about the benefits $(2 \mathrm{E}$ and $8 \mathrm{~B})$, incompatibility with clinical focus and prioritisation $(6 \mathrm{~K}$ and $7 \mathrm{G}$ ) and lack of resources and consideration in early healthcare project phases (4C and 9E). A common thread is again evident corresponding similarly with architecture and design issues. This concurs with other findings indicating that poor design decisions and execution and lack of planning may greatly restrict the available scope of positive healthcare experiences. ${ }^{32}{ }^{40}$ Inappropriate contextual and environmental factors may create adverse reactions and impact negatively on the quality of healthcare experiences. ${ }^{39}$ Although experts identified real barriers, they 
Table 2 Highest-ranked items in Q4 ( $n=32 ; 84 \%)$

\section{Ranking \\ (category, \\ see box)}

Item description

\begin{tabular}{lll} 
Q3, $\mathrm{n}=31$ & Q4, $\mathrm{n}=32$ & Q4, $\mathrm{n}=32$ \\
\hline Total & Median $($ IQR $)$ & $\% \geq 7$
\end{tabular}

Opportunities

1 (C)

Window views from clinical areas onto nature, garden, sea, sky, weather, 140

$10(9-10)$

97

people watching, greenery, trees, outside world, daylight, night sky,

escape, movement, change, without glare, attention to privacy (one-way

views)

\begin{tabular}{|c|c|c|c|c|}
\hline 2 (A) & $\begin{array}{l}\text { Accessible outdoor settings, gardens and courtyards: easy and effortless } \\
\text { access, automatic doors, nearby, some areas with high visibility, close } \\
\text { proximity to clinical assistance, remove barriers and thresholds, available } \\
\text { for patients, carers and staff }\end{array}$ & 253 & $10(9-10)$ & 100 \\
\hline $3(E)$ & $\begin{array}{l}\text { Physical exercise adapted to patient requirements: stroll garden, walking } \\
\text { paths with points of interest and distance markers (plant species, } \\
\text { medicinal plants), meandering trails, resting points, exercise opportunity } \\
\text { for staff, nature walks, mindful walking, mobility and balance training, } \\
\text { gardening tasks, assisted walking, nature exercise rooms, labyrinths }\end{array}$ & 101 & $9(7-10)$ & 91 \\
\hline 4 (B) & $\begin{array}{l}\text { Appropriate safety measures and surface materials for limited mobility: } \\
\text { handrails, smooth paved paths, ramps rather than steps, colour } \\
\text { contrasting curbing along pathways }\end{array}$ & 35 & $9(7-10)$ & 90 \\
\hline 5 (D) & $\begin{array}{l}\text { Educate healthcare team, management, patients, designers, policy and } \\
\text { decision makers about value, benefits and appropriate implementation of } \\
\text { nature-based opportunities }\end{array}$ & 70 & $8(7-10)$ & 94 \\
\hline $6(C)$ & $\begin{array}{l}\text { Design for privacy: zoning, screening, semienclosed spaces, restful, } \\
\text { contemplative and solitary spaces, some outdoor spaces shielded from } \\
\text { inside views, separate but nearby spaces for staff to retreat (away from } \\
\text { patients and workplace) }\end{array}$ & 75 & 8 (7-9) & 88 \\
\hline $7(\mathrm{G})$ & $\begin{array}{l}\text { Design proposal needs to address repair and maintenance requirements } \\
\text { of nature-based features within available maintenance budgets (easy to } \\
\text { maintain). Tasks to be carried out by skilled professionals }\end{array}$ & 61 & $8(7-9)$ & 84 \\
\hline $8(C)$ & $\begin{array}{l}\text { Protection from adverse weather conditions (sun, shade, high/low } \\
\text { temperatures) and unpleasant stimulation (overpowering scents, noise, } \\
\text { loud sounds, toxic plants, clutter) }\end{array}$ & 40 & 8 (7-9) & 84 \\
\hline $9(\mathrm{~F})$ & $\begin{array}{l}\text { Socialising: range of seating options, gathering and communal spaces, } \\
\text { BBQ area, children play areas, semiprivate enclosures for personal } \\
\text { conversations }\end{array}$ & 65 & $8(7-9)$ & 84 \\
\hline $10(\mathrm{C})$ & $\begin{array}{l}\text { Indoor design to maximise use of biophilic elements: natural materials, } \\
\text { natural colours, airflow (including windows that open safely) and natural } \\
\text { light }\end{array}$ & 41 & 8 (7-8) & 88 \\
\hline
\end{tabular}

Barriers

\begin{tabular}{|c|c|c|c|c|}
\hline $1(\mathrm{~A})$ & $\begin{array}{l}\text { Building design and site constraints, missed opportunities: layout, } \\
\text { building orientation, surrounding views, lack of available space were not } \\
\text { considered in planning and development phase }\end{array}$ & 194 & $9(8-10)$ & 91 \\
\hline $2(\mathrm{E})$ & $\begin{array}{l}\text { Decision makers, management and administration often lack knowledge } \\
\text { and/or awareness about benefits of nature engagement }\end{array}$ & 175 & $9(8-10)$ & 94 \\
\hline 3 (D) & $\begin{array}{l}\text { Inaccessibility: heavy, locked doors, no electronic door opener, barriers, } \\
\text { thresholds, doorways and pathways too narrow for wheelchair or gurney } \\
\text { access or for two wheelchairs to pass, too wide paver joints become } \\
\text { tripping hazards, insufficient seating, co-opted as smoking areas, access } \\
\text { for the very sick and frail not considered }\end{array}$ & 141 & $9(7-10)$ & 91 \\
\hline $4(C)$ & $\begin{array}{l}\text { Cost and resource allocation: cost for routine repair and maintenance, } \\
\text { staff and volunteer time, acquiring indoor equipment (screens, virtual } \\
\text { reality, AV), lack of funding, often based on fundraising and grants }\end{array}$ & 179 & $9(8-10)$ & 91 \\
\hline
\end{tabular}

Continued 


\begin{tabular}{|c|c|c|c|c|}
\hline $\begin{array}{l}\text { Ranking } \\
\text { (category, } \\
\text { see box) }\end{array}$ & Item description & Q3, $n=31$ & Q4, $n=32$ & Q4, $n=32$ \\
\hline & & Total & Median (IQR) & $\% \geq 7$ \\
\hline $5(F)$ & $\begin{array}{l}\text { Inappropriate design choices and execution: limited greenery, cold and } \\
\text { stark, too much hardscape (concrete, glare), uncomfortable seating, too } \\
\text { demanding, complex, static or boring environments, insufficient shading, } \\
\text { materials too hot to the touch, structures/sculptures that cast odd } \\
\text { shadows }\end{array}$ & 53 & $9(7-10)$ & 91 \\
\hline $6(\mathrm{~K})$ & $\begin{array}{l}\text { Healthcare facilities design often guided by clinical functionality, } \\
\text { efficiency, cost restrictions and/or habitual practice, not necessarily the } \\
\text { patient perspective/experience }\end{array}$ & 99 & $8(7-10)$ & 94 \\
\hline $7(\mathrm{G})$ & $\begin{array}{l}\text { Mainstream values (decision makers) do not prioritise nature-based } \\
\text { opportunities or 'design thinking' }\end{array}$ & 23 & $8(7-9)$ & 91 \\
\hline $10(F)$ & $\begin{array}{l}\text { Inauthenticity of nature-based design elements: fake plants, fake scents, } \\
\text { tokenistic, corporate design ('cutting edge' award-seeking designs) }\end{array}$ & 58 & $8(7-10)$ & 81 \\
\hline
\end{tabular}

simultaneously suggested useful response to these challenges. Highlighted, in particular, is the importance of planning in regard to healthcare design and development, healthcare policy and messaging and clinical practice. During early stage planning phases, considerations such as site layout, window views, integrated hospital courtyards and biophilic design elements may be incorporated without great cost burden and can be designed with maintenance budgets in mind. Lack of foresight in healthcare design projects, as detected in the findings, can result in suboptimal design choices and forgo potential user benefits. The findings emphasise need for education and awareness about the essential value, demand and appropriate implementation of nature-based initiatives. The patient perspective (6K) may usefully guide decision making about designing for experiences of value to the end user. ${ }^{42}$

These findings combined are not surprising and concur with existing health-nature research, which report various biopsychosocial benefits from the interchanges with nature of patients with cancer. ${ }^{11-13} 3243$ Psycho-oncology literature further corroborates the psychological importance of oncology environment design. ${ }^{44} 45$ Such contextual features powerfully communicate symbols of care and caring and may meaningfully affect patients' lived healthcare experiences and perceptions. ${ }^{39}$

\section{Limitations}

Knowledge generated by a Delphi panel represents input from a select sample of experts at a given time and may differ when involving other experts. To mitigate this limitation, an acceptable panel size was used ${ }^{21}$ involving a heterogeneous sample of experts with relevant professional and academic backgrounds to collect a diverse range of viewpoints about the topic. Furthermore, there may be variation in levels of experience and knowledge about the specific topic despite panellists reporting an average of 20.5 years of expert experience. The findings represent contributions from a specific expert sample, which may differ when involving other experts. The panel's exposure to patients' own recommendations created a common basis for this investigation and helped to define its topical parameters and stimulated their novel thinking on the topic.

The recommendations generated in this study describe experts' key concerns relating to nature-based care opportunities in oncology contexts; the findings cannot be seen as prescriptive for the design of all cancer care environments and services across different settings and patient groups. Although the panel included experts with varying backgrounds who represented seven different countries, the findings likely express a Westernised perspective and may not apply to other contexts. Furthermore, participants' geographic locations in regard to their native, natural environments and climatic conditions may have influenced input in this study. It bears mention that more than half of the participants originated from Oceania (Australia (19) and New Zealand (2)), which may have resulted in biased recommendations. The appropriateness and adoption of nature-based care scenarios need to be assessed based on country- and patient-specific standards and guidelines to guarantee safe practices and best outcomes. Lastly, although the study achieved a panel of mixed experts, the sample included a greater number of academics and HPs than HMs and ADs. The type of expert input needs to be considered when appraising the findings. Future research may further investigate reasons for non-participation of certain expert groups and evaluate the significance of their contributions or lack thereof. 


\section{Practical implications}

The recommendations (table 2) developed in this study reflect key concerns shared across stakeholder groups and can be easily and quickly accessed by relevant parties involved in the design and development of oncology settings and care services. They can be used to:

- guide healthcare designers and architects when planning and designing new cancer care settings and improving existing ones;

- inform and educate healthcare practice about appropriate and safe nature-based care opportunities;

- extend established cancer care services by using embedded or readily available resources (eg, improving access to hospital courtyards, maximising window views, integrating physical exercise programme in available hospital outdoor settings);

- provide key items as quality markers for establishing and assessing nature-based care opportunities in oncology contexts.

\section{CONCLUSION}

To our best knowledge, this study represents the first international and cross-disciplinary effort to generate expert recommendations related to the integration of naturebased care opportunities in oncology contexts. These recommendations elucidate as yet unexplored healthcare responses that use the care setting itself, its context and ambient features to improve patient outcomes. Reflecting patient and expert perspectives, these nature-based care recommendations have particular relevance for healthcare, but importantly, they advocate for often unvoiced healthcare needs and human experiences, which can add great value to oncology patient experiences. The findings provide insight into a new sphere of design and intervention for humanising oncology environments and improving daily healthcare experiences of people affected by cancer and those working in the cancer care setting.

Contributors SB, CCC and PS conceived the study and collaborated on the initial protocol and study materials. SB analysed and interpreted the data followed by inter-rater discussion with CCC and PS, who helped interpret the data and critically revised the manuscript. SB is the guarantor of the study.

Funding SB was supported by an Australian Government Research Training Program Scholarship throughout the study period.

Competing interests None declared.

Ethics approval Peter MacCallum Human Research Ethics Committee.

Provenance and peer review Not commissioned; externally peer reviewed.

Data sharing statement № additional data are available.

Open Access This is an Open Access article distributed in accordance with the Creative Commons Attribution Non Commercial (CC BY-NC 4.0) license, which permits others to distribute, remix, adapt, build upon this work non-commercially, and license their derivative works on different terms, provided the original work is properly cited and the use is non-commercial. See: http://creativecommons.org/ licenses/by-nc/4.0/

(C) Article author(s) (or their employer(s) unless otherwise stated in the text of the article) 2017. All rights reserved. No commercial use is permitted unless otherwise expressly granted.

\section{REFERENCES}

1. Maller C, et al. Healthy nature healthy people: 'contact with nature' as an upstream health promotion intervention for populations. Health Promot Int 2006;21:45-54.

2. Mcsweeney J, Rainham D, Johnson SA, et al. Indoor nature exposure (INE): a health-promotion framework. Health Promot Int 2015;30:126-39.

3. Spring JA. Design of evidence-based gardens and garden therapy for neurodisability in Scandinavia: data from 14 sites. Neurodegener Dis Manag 2016;6:87-98.

4. WÃahrborg P, Petersson I, Grahn P. Nature-assisted rehabilitation for reactions to severe stress and/or depression in a rehabilitation garden: long-term follow-up including comparisons with a matched population-based reference cohort. J Rehabil Med 2014;46:271-6.

5. Stewart B, Wild C. World cancer report: International Agency for Research on Cancer, World Health Organization, 2014.

6. Herzog TR, Chen HC, Primeau JS. Perception of the restorative potential of natural and other settings. J Environ Psychol 2002;22:295-306.

7. Antonovsky A. The salutogenic model as a theory to guide health promotion. Health Promot Int 1996;11:11-18.

8. Sanson-Fisher R, Girgis A, Boyes A, et al. The unmet supportive care needs of patients with cancer. Cancer 2000;88:226-37.

9. Zabora JR, Blanchard CG, Smith ED, et al. Prevalence of psychological distress among cancer patients across the disease continuum. J Psychosoc Oncol 1997;15:73-87.

10. Ashbury FD, Findlay H, Reynolds B, et al. A Canadian survey of cancer patients' experiences: are their needs being met? J Pain Symptom Manage 1998;16:298-306.

11. Rowlands J, Noble S. How does the environment impact on the quality of life of advanced cancer patients? A qualitative study with implications for ward design. Palliat Med 2008;22:768-74.

12. Blair CK, Madan-Swain A, Locher JL, et al. Harvest for health gardening intervention feasibility study in cancer survivors. Acta Oncol 2013;52:1110-8.

13. Cimprich B, Ronis DL. An environmental intervention to restore attention in women with newly diagnosed breast cancer. Cancer Nurs 2003;26:284-92.

14. Sherman SA, Varni JW, Ulrich RS, et al. Post-occupancy evaluation of healing gardens in a pediatric cancer center. Landsc Urban Plan 2005;73:167-83.

15. Blaschke $S, O^{\prime}$ Callaghan $C C$, Schofield P, et al. Cancer patients' experiences with nature: Normalizing dichotomous realities. Soc Sci Med 2017;172:107-14.

16. Blaschke $S$. The role of nature in cancer patients' lives: a systematic review and qualitative meta-synthesis. BMC Cancer 2017; $17: 370$.

17. Blaschke S, O'Callaghan CC, Schofield P. Identifying opportunities for nature engagement in cancer care practice and design: protocol for four-round modified electronic Delphi. BMJ Open 2017;7:e013527.

18. Schmidt RC. Managing Delphi surveys using nonparametric statistical techniques*. Decision Sci 1997;28:763-74.

19. Tetzlaff JM, Moher D, Chan A-W. Developing a guideline for clinical trial protocol content: Delphi consensus survey. Trials 2012;13:176.

20. Chan A-W, Tetzlaff JM, Gotzsche PC, et al. SPIRIT 2013 explanation and elaboration: guidance for protocols of clinical trials. BMJ 2013;346:e7586.

21. Mullen PM. Delphi: myths and reality. J Health Organ Manag 2003;17:37-52

22. Keeney S, Hasson F, McKenna HP. A critical review of the Delphi technique as a research methodology for nursing. Int J Nurs Stud 2001;38:195-200.

23. Schmidt R, Lyytinen K, Mark Keil PC. Identifying software project risks: an international Delphi study. JMIS 2001;17:5-36.

24. Okoli C, Pawlowski SD. The Delphi method as a research tool: an example, design considerations and applications. Inf Manage 2004;42:15-29.

25. Linstone HA, Turoff M. The Delphi method: techniques and applications. MA: Addison-Wesley Reading, 1975.

26. Hasson F, Keeney S. Enhancing rigour in the Delphi technique research. Technol Forecast Soc Change 2011;78:1695-704.

27. Collins D. Pretesting survey instruments: an overview of cognitive methods. Qual Life Res 2003;12:229-38.

28. Strauss A, Corbin JM. Basics of qualitative research: qrounded theory procedures and techniques Sage Publications, Inc, 1990.

29. IBM C. IBM SPSS Statistics for Macintosh, version 23. Armonk, NY: IBM Corp 2013.

30. Diamond IR, Grant RC, Feldman BM, et al. Defining consensus: a systematic review recommends methodologic criteria for reporting of Delphi studies. J Clin Epidemiol 2014;67:401-9. 
31. Ray HA, Verhoef MJ. Dragon boat racing and health-related quality of life of breast cancer survivors: a mixed methods evaluation. BMC Complement Altern Med 2013;13:205.

32. Butterfield. Resilient places? healthcare gardens and the maggie's centres. Doctoral thesis, Falmouth University 2014.

33. Spees CK, Joseph A, Darragh A, et al. Health behaviors and perceptions of cancer survivors Harvesting at an urban garden. Am J Health Behav 2015;39:257-66.

34. Unruh AM, Smith N, Scammell C. The occupation of gardening in life-threatening illness: a qualitative pilot project. Can J Occup Ther 2000;67:70-7.

35. English J, Wilson K, Keller-Olaman S. Health, healing and recovery: therapeutic landscapes and the everyday lives of breast cancer survivors. Soc Sci Med 2008;67:68-78.

36. Unruh A, Hutchinson S. Embedded spirituality: gardening in daily life and stressful life experiences. Scand J Caring Sci 2011;25:567-74.

37. Minton C, Batten L. Rethinking the intensive care environment: considering nature in nursing practice. J Clin Nurs 2016;25:269-77.

38. Fleming R, Kelly F, Stillfried G. 'I want to feel at home': establishing what aspects of environmental design are important to people with dementia nearing the end of life. BMC Palliat Care 2015;14:26.
39. Edvardsson D, Sandman PO, Rasmussen B. Caring or uncaring - meanings of being in an oncology environment. J Adv Nurs 2006;55:188-97.

40. Ulrich RS, Zimring C, Zhu X, et al. A review of the research literature on evidence-based healthcare design. HERD 2008;1:61-125.

41. van den Berg AE. Health impacts of healing environments: a review of evidence for benefits of nature, daylight, fresh air, and quiet in healthcare settings. Dodewaard (NL): Van Eck \& Oosterink, 2005

42. Tsianakas V, Robert G, Maben J, et al. Implementing patient-centred cancer care: using experience-based co-design to improve patient experience in breast and lung cancer services. Support Care Cancer 2012:20:2639-47.

43. Ray H, Jakubec SL. Nature-based experiences and health of cancer survivors. Complement Ther Clin Pract 2014;20:188-92.

44. Edvardsson JD, Sandman P-O, Rasmussen BH. Sensing an atmosphere of ease: a tentative theory of supportive care settings. Scand J Caring Sci 2005;19:344-53.

45. Andritsch E, Stöger H, Bauernhofer T, et al. The ethics of space, design and color in an oncology ward. Palliat Support Care 2013;11:215-21. 